PROCEEDINGS OF THE

AMERICAN MATHEMATICAL SOCIETY

Volume 135, Number 10, October 2007, Pages 3329-3340

S 0002-9939(07)08857-0

Article electronically published on June 20, 2007

\title{
FINITE PROPAGATION SPEED AND KERNEL ESTIMATES FOR SCHRÖDINGER OPERATORS
}

\author{
CHRISTIAN REMLING
}

(Communicated by Joseph A. Ball)

\begin{abstract}
We point out finite propagation speed phenomena for discrete and continuous Schrödinger operators and discuss various types of kernel estimates from this point of view.
\end{abstract}

\section{INTRODUCTION}

In this note, finite propagation speed phenomena associated with continuous Schrödinger operators

$$
(H u)(x)=-\Delta u(x)+V(x) u(x)
$$

on $L_{2}\left(\mathbb{R}^{d}\right)$ and their discrete analogs

$$
(H u)(n)=\sum_{|m-n|_{1}=1} u(m)+V(n) u(n),
$$

acting on $\ell_{2}\left(\mathbb{Z}^{d}\right)$, are pointed out. We believe that these observations lead to a very transparent, non-technical and elegant treatment of various topics.

It is instructive to look at the one-dimensional half-line problems for a moment. So, consider the operators from (1.1) and (1.2), acting on $L_{2}(0, \infty)$ and $\ell_{2}(\mathbb{N})$, respectively. Let $\rho$ be the standard spectral measure; it can be obtained from the Weyl circle construction (see, for example, [4). Then, in the discrete case, the moments $\int \lambda^{n} d \rho(\lambda)$ are a very important object, and experience has shown that a similar role is played by the function

$$
\phi(t)=\int \cos t \sqrt{\lambda} d \rho(\lambda)-2 \delta(t)
$$

in the continuous case. (It can be shown that this formula, suitably interpreted, indeed defines an absolutely continuous function. See, for example, 12.) In other words, there seems to be something special about the functions $\lambda^{n}$ and $\cos t \sqrt{\lambda}$, respectively. As we will see in Section 2, the unifying theme is finite propagation speed, and this in fact works in any dimension.

We will illustrate our basic observations (see Lemmas 2.1 and 2.2 below) further by using them to discuss the following two problems:

Received by the editors January 27, 2006 and, in revised form, July 18, 2006.

2000 Mathematics Subject Classification. Primary 81Q10, 35J10, 47B39, 47F05.

Key words and phrases. Schrödinger operator, finite propagation speed, kernel estimates.

(C)2007 American Mathematical Society Reverts to public domain 28 years from publication 
1. Let $f$ be a bounded smooth function on the spectrum of $H$. Try to estimate $\left\langle\varphi_{1}, f(H) \varphi_{2}\right\rangle$ in terms of the separation of the supports of $\varphi_{1}$ and $\varphi_{2}$.

2. Suppose that $V$ is known on $B_{R}(0)=\left\{x \in \mathbb{R}^{d}:|x|<R\right\}$ and $\varphi$ is supported near zero. What can one then say about $\langle\varphi, f(H) \varphi\rangle$ ?

Both problems have been studied before by other methods, the first one in fact quite extensively (see [1] and the references cited therein). The sample results we will prove in Sections 3 and 4 below are quite similar to what had been known before. Actually, Corollary 5.3 below does seem to improve results from [1, but that is definitely not the main point in this context. Rather, what we are trying to emphasize here is the realization that finite propagation speed phenomena are at the heart of the matter, and things become very transparent if this point of view is adopted. This basic idea might be useful in other situations too.

A piece of closely related work is [2], where finite propagation speed methods are used to estimate kernels of functions of the Laplace-Beltrami operator on Riemannian manifolds. In [16], these techniques are used to estimate heat kernels. Finally, see [6, 13] for work on problem 2, and it may also be interesting to take a look at [9] or [11, Sect. XI.11] for the use of wave phenomena in scattering theory.

\section{Finite propagation SPeED}

We make the following basic assumptions: In the continuous case, we assume that $H$ is essentially self-adjoint on $C_{0}^{\infty}\left(\mathbb{R}^{d}\right)$ and bounded below. A popular sufficient condition for this is $V \in L_{2, l o c}$ and $V_{-} \in K_{d}$, the Kato class ( $V_{-}$denotes the negative part of $V)$. See [17, Sect. 3]. Conversely, the fact that $C_{0}^{\infty}\left(\mathbb{R}^{d}\right)$ functions are in the domain of $H$ implies that $V \in L_{2, l o c}$.

In the discrete case, we assume that $V$ is bounded. It is then of course automatic that $H$ generates a bounded, self-adjoint operator on $\ell_{2}\left(\mathbb{Z}^{d}\right)$.

Let us begin with the discrete case. Here the basic lemma is extremely simple.

Lemma 2.1 (Finite propagation speed - discrete case). Consider the operator $H$ from (1.2).

(a) Let $\varphi_{1}, \varphi_{2} \in \ell_{2}\left(\mathbb{Z}^{d}\right)$, and let $R$ be the distance, measured in the $|\cdot|_{1}$ norm on $\mathbb{Z}^{d}$, between the supports of $\varphi_{1}$ and $\varphi_{2}$. Then

$$
\left\langle\varphi_{1}, H^{n} \varphi_{2}\right\rangle=0 \quad \text { for } n=0,1, \ldots, R-1 .
$$

(b) Consider two (bounded) potentials $V_{1}, V_{2}$ and the corresponding Schrödinger operators $H_{1}, H_{2}$, let $\varphi \in \ell_{2}\left(\mathbb{Z}^{d}\right)$, and, similarly to the definition in part (a), let

$$
R=\operatorname{dist}\left(\operatorname{supp} \varphi, \operatorname{supp}\left(V_{1}-V_{2}\right)\right) \text {. }
$$

Then

$$
\left\langle\varphi, H_{1}^{n} \varphi\right\rangle=\left\langle\varphi, H_{2}^{n} \varphi\right\rangle \quad \text { for } n=0,1, \ldots, 2 R .
$$

Proof. It is clear from the form of $H$ that the support of $H \psi$ is contained in a 1-neighborhood (in the $|\cdot|_{1}$ norm) of the support of $\psi$. Repeatedly applying this gives (a).

It also follows that in the situation of part (b), $H_{1}^{n} \varphi=H_{2}^{n} \varphi$ for $n \leq R$ since of course $H_{1} \psi$ can only be different from $H_{2} \psi$ if $\psi$ is non-zero at one of the points where $V_{1} \neq V_{2}$. Now it may happen that $\left(H_{1}^{R+1} \varphi\right)(x) \neq\left(H_{2}^{R+1} \varphi\right)(x)$ for some $x \in \mathbb{Z}^{d}$, but, according to what has just been observed, only at points $x$ from the support of $V_{1}-V_{2}$, and thus it takes at least $R$ more steps to get back to the support of $\varphi$ (formally, we could in fact apply part (a) to see this). 
By the spectral theorem, part (b) also says that

$$
\int_{\mathbb{R}} \lambda^{n} d \rho_{1}(\lambda)=\int_{\mathbb{R}} \lambda^{n} d \rho_{2}(\lambda)
$$

for $n=0,1, \ldots, 2 R$. Here, $\rho_{j}$ is the spectral measure for $H_{j}$ and $\varphi$. As already discussed in the Introduction, the appropriate continuous substitutes for the powers $\lambda^{n}$ seem to be the functions $\cos t \sqrt{\lambda}$, at least for one-dimensional problems. See also 13 for further background information.

This suggests the following continuous analog of Lemma 2.1. Since we are assuming that $H$ from (1.1) is bounded below, $\cos t \sqrt{\lambda}$ is a bounded function on the spectrum of $H$. Also, this function clearly does not depend on a choice of the square root and is in fact entire in $\lambda$.

Lemma 2.2 (Finite propagation speed - continuous case). Consider the operator $H$ from (1.1).

(a) Let $\varphi_{1}, \varphi_{2} \in L_{2}\left(\mathbb{R}^{d}\right)$ and define

$$
R=\operatorname{dist}\left(\operatorname{supp} \varphi_{1}, \operatorname{supp} \varphi_{2}\right) \text {. }
$$

Then

$$
\left\langle\varphi_{1}, \cos t \sqrt{H} \varphi_{2}\right\rangle=0 \quad \text { for }|t| \leq R .
$$

(b) Consider two potentials $V_{1}, V_{2}$, let $\varphi \in L_{2}\left(\mathbb{R}^{d}\right)$ and define

$$
R=\operatorname{dist}\left(\operatorname{supp} \varphi, \operatorname{supp}\left(V_{1}-V_{2}\right)\right) \text {. }
$$

Then

$$
\left\langle\varphi, \cos t \sqrt{H_{1}} \varphi\right\rangle=\left\langle\varphi, \cos t \sqrt{H_{2}} \varphi\right\rangle \text { for }|t| \leq 2 R .
$$

Proof. By a routine approximation argument, we may and will assume that all functions $V$ and $\varphi$ are in $C_{0}^{\infty}\left(\mathbb{R}^{d}\right)$. Indeed, since the operators $\cos t \sqrt{H}$ are bounded, we can certainly approximate the $\varphi \mathrm{s}$ in $L_{2}\left(\mathbb{R}^{d}\right)$ by smooth functions, and we then have convergence of the scalar products we are interested in. We then pick $V_{n} \in$ $C_{0}^{\infty}\left(\mathbb{R}^{d}\right)$ so that $H_{n}=-\Delta+V_{n} \rightarrow H$ in strong resolvent sense. This is possible by [10, Theorem VIII.25(a)]. But then also $\cos t \sqrt{H_{n}} \rightarrow \cos t \sqrt{H}$ strongly, by [10, Theorem VIII.20(b)]. Finally, we can do these approximations in such a way that $\lim \inf R_{n} \geq R$ for the separations $R$ defined in the lemma.

Now let $u=\cos t \sqrt{H} \varphi_{2}$. Then $u$ solves

$$
u_{t t}=-H u, \quad u(0)=\varphi_{2}, \quad u_{t}(0)=0 .
$$

Originally, this needs to be interpreted as an equation for functions of $t$ taking values in $L_{2}\left(\mathbb{R}^{d}\right)$, but $V$ and $\varphi$ are smooth now, so the regularity results for weak solutions of (generalized) wave equations actually show that $u \in C^{\infty}\left(\mathbb{R}^{d} \times \mathbb{R}\right)$ and

$$
u_{t t}(x, t)-\Delta u(x, t)=-V(x) u(x, t)
$$

holds pointwise. Compare [5, Theorem 7.2.7].

We now need the following classical fact.

Lemma 2.3 (Finite propagation speed - wave equation). Suppose $u \in C^{\infty}$ solves (2.1) and $u(x, 0)=u_{t}(x, 0)=0$ for $\left|x-x_{0}\right|<r$. Then $u \equiv 0$ on $\left|x-x_{0}\right| \leq r-|t|$.

Proof of Lemma 2.3. We will use an energy estimate. We can assume that $u$ is real valued and $x_{0}=0$. Define

$$
E(t)=\int_{B(r-t)}\left(u^{2}+u_{t}^{2}+|\nabla u|^{2}\right) d x
$$


here, $B(q)=\left\{x \in \mathbb{R}^{d}:|x|<q\right\}$ denotes the ball of radius $q$. Also, let $S(q)$ be the sphere $S(q)=\{x:|x|=q\}$, and write $\sigma$ for the surface measure on $S(q)$. We will now compute $E^{\prime}$ :

$$
E^{\prime}=-\int_{S(r-t)}\left(u^{2}+u_{t}^{2}+|\nabla u|^{2}\right) d \sigma+2 \int_{B(r-t)}\left(u u_{t}+u_{t} u_{t t}+\nabla u \cdot \nabla u_{t}\right) d x .
$$

An integration by parts and use of (2.1) allow us to write the second integral in the form

$$
2 \int_{B(r-t)}(1-V) u u_{t} d x+2 \int_{S(r-t)} u_{t} \mathbf{n} \cdot \nabla u d \sigma
$$

where $\mathbf{n}$ is the outer normal unit vector on the sphere. By the Cauchy-Schwarz inequality, the last term can be estimated by

$$
2\left(\int_{S(r-t)} u_{t}^{2} d \sigma\right)^{1 / 2}\left(\int_{S(r-t)}|\nabla u|^{2} d \sigma\right)^{1 / 2} .
$$

Putting things together, we thus see that

$$
E^{\prime} \leq 2 \int_{B(r-t)}(1+|V|)\left|u u_{t}\right| d x .
$$

Now $V$ is bounded, so a final application of the Cauchy-Schwarz inequality shows that

$$
E^{\prime} \leq C\left(\int_{B(r-t)} u^{2} d x\right)^{1 / 2}\left(\int_{B(r-t)} u_{t}^{2} d x\right)^{1 / 2} \leq C E .
$$

Since $E(0)=0$, Gronwall's Lemma implies that $E \equiv 0$. This proves the claim (for $t>0$, but $u(x,-t)$ satisfies the same equation).

Part (a) of Lemma 2.2 now follows easily: If $x_{0}$ is an arbitrary point from the support of $\varphi_{1}$, then, by assumption, $\varphi_{2}(x)=0$ for all $\left|x-x_{0}\right|<R$. Lemma 2.3 thus shows that $\left(\cos t \sqrt{H} \varphi_{2}\right)(x)=0$ if $\left|x-x_{0}\right| \leq R-|t|$. In particular, $\left(\cos t \sqrt{H} \varphi_{2}\right)\left(x_{0}\right)=0$ for $|t| \leq R$. Since $x_{0} \in \operatorname{supp} \varphi_{1}$ was arbitrary, it follows that $\left\langle\varphi_{1}, \cos t \sqrt{H} \varphi_{2}\right\rangle=0$ for these $t$, as claimed.

To prove part (b), let $u_{j}=\cos t \sqrt{H_{j}} \varphi$. The argument is similar to the proof of Lemma 2.1(b): It takes $T$ units of time to reach points where $V_{1} \neq V_{2}$ (starting from the support of $\varphi$ ), and then another time span $T$ to get back to the support of $\varphi$. To write this down more formally, first note that Lemma 2.3 shows that $u_{j}(x, t)=0$ whenever $\operatorname{dist}(x, \operatorname{supp} \varphi)>|t|$. This shows that for $|t| \leq R$, the difference function $u=u_{2}-u_{1}$ solves

$$
u_{t t}-\Delta u=-V_{1} u
$$

for all $x \in \mathbb{R}^{d}$. Indeed, if $\operatorname{dist}(x, \operatorname{supp} \varphi)<R$, (2.2) follows from the fact that then $V_{1}(x)=V_{2}(x)$, and if $\operatorname{dist}(x, \operatorname{supp} \varphi)>R$, then, as we have just seen, $u_{1}(x, t)=$ $u_{2}(x, t)=0$ for $|t| \leq R$.

Since $u(0)=u_{t}(0)=0$, Lemma 2.3, applied to (2.2), shows that $u \equiv 0$ for $|t| \leq R$. As argued above, if $\operatorname{dist}(x, \operatorname{supp} \varphi)<R$, then (2.2) in fact holds for all $t$, so a final application of Lemma 2.3 (to $u(t \pm R)$ ) shows that $u(t)=0$ on the support of $\varphi$ for $|t| \leq 2 R$, as desired. 


\section{DECAY OF KERNELS}

We begin our discussion of applications of Lemmas 2.1 and 2.2 with the first problem mentioned in the Introduction, and we first deal with the discrete case. We will use the following classical result from approximation theory:

Theorem 3.1 (Jackson). Let $f \in C^{\infty}[-1,1]$. Then for every $n \in \mathbb{N}$ and $R \geq n$, there exist polynomials $p_{R}$, $\operatorname{deg} p_{R} \leq R$, so that

$$
\left\|f-p_{R}\right\|_{\infty} \leq\left\|f^{(n+1)}\right\|_{1}\left(\frac{5}{R+1}\right)^{n} .
$$

See [3, Sect. 4.6] or [14, Sect. 1.1] for quick proofs (the second reference has bigger constants) and [18] for a more comprehensive discussion of these issues, including optimal results. To obtain the result in the form stated above, use the inequality

$$
N(N-1) \cdots(N-k+1) \geq\left(\frac{N}{e}\right)^{k},
$$

and note that the modulus of continuity of a function $g$, defined as $\omega(g, \delta)=$ $\sup _{|x-y| \leq \delta}|g(x)-g(y)|$, can obviously be estimated by $\omega(g, \delta) \leq\left\|g^{\prime}\right\|_{1}$ if $g$ is differentiable. To prove (3.1), one can use a version of Stirling's formula for $n$ ! with explicit error estimates, or simply estimate the logarithm of the left-hand side:

$$
\sum_{n=N-k+1}^{N} \ln n \geq \int_{N-k}^{N} \ln x d x=N \ln N-(N-k) \ln (N-k)-k \geq k(\ln N-1),
$$

as claimed.

We'll use the following notation: For $x \in \mathbb{Z}^{d}$, let $\delta_{x}$ be the unit vector located at $x$ (so $\delta_{x}(x)=1, \delta_{x}(y)=0$ if $\left.y \neq x\right)$. Moreover, $\sigma(H)$ denotes, as usual, the spectrum of $H$. The estimates we are about to prove will depend on the diameter of the spectrum.

Theorem 3.2. Suppose that $\sigma(H) \subset[a, b]$ and $f \in C^{\infty}[a, b]$. Then, for $n=$ $1,2, \ldots,|x-y|_{1}-1$,

$$
\left|\left\langle\delta_{x}, f(H) \delta_{y}\right\rangle\right| \leq\left\|f^{(n+1)}\right\|_{L_{1}(a, b)}\left(\frac{5(b-a)}{2|x-y|_{1}}\right)^{n} .
$$

Proof. Given Lemma 2.1, the proof is straightforward: Let

$$
s=\frac{2 \lambda-a-b}{b-a}, \quad g(s)=f\left(\frac{a+b}{2}+\frac{b-a}{2} s\right),
$$

and approximate $g$ on $[-1,1]$ by a polynomial of degree $\leq R-1$, with $R \equiv|x-y|_{1}$ :

$$
g(s)=p_{R-1}(s)+e_{R-1}(s), \quad-1 \leq s \leq 1 .
$$

By Lemma 2.1(a),

hence

$$
\left\langle\delta_{x}, p_{R-1}\left(\frac{2 H-a-b}{b-a}\right) \delta_{y}\right\rangle=0
$$

$$
\left|\left\langle\delta_{x}, f(H) \delta_{y}\right\rangle\right| \leq \max _{-1 \leq s \leq 1}\left|e_{R-1}(s)\right| .
$$

By Theorem 3.1 $p_{R-1}$ can be chosen so that

$$
\left|e_{R-1}(s)\right| \leq\left(\frac{5}{R}\right)^{n}\left\|g^{(n+1)}\right\|_{L_{1}(-1,1)} .
$$


Since $\left\|g^{(n+1)}\right\|_{L_{1}(-1,1)}=\left(\frac{b-a}{2}\right)^{n}\left\|f^{(n+1)}\right\|_{L_{1}(a, b)}$, the asserted estimate follows.

The discussion of the continuous case proceeds along similar lines. We will find it convenient to assume that $H \geq 0$, where now $H$ is the continuous Schrödinger operator from (1.1); the general case ( $H$ semibounded below) can of course be reduced to this situation by adding a suitable constant to $H$ (respectively, $V$ ).

Theorem 3.3. Assume that $H \geq 0$. Let $f \in C^{\infty}[0, \infty), \varphi_{1}, \varphi_{2} \in L_{2}\left(\mathbb{R}^{d}\right)$, and define

$$
R=\operatorname{dist}\left(\operatorname{supp} \varphi_{1}, \operatorname{supp} \varphi_{2}\right) \text {. }
$$

Also, let $g(k)=f\left(k^{2}\right)$ and assume that $g^{(n)} \in L_{1}(0, \infty)$ for all $n \geq 0$. Then, for each $n \in \mathbb{N}$, we have that

$$
\left|\left\langle\varphi_{1}, f(H) \varphi_{2}\right\rangle\right| \leq \frac{2\left\|\varphi_{1}\right\|\left\|\varphi_{2}\right\|}{\pi n} \frac{\left\|g^{(n+1)}\right\|_{L_{1}(0, \infty)}}{R^{n}} .
$$

Proof. Expand $f$ in terms of the cosine functions from Lemma 2.2

$$
\begin{aligned}
f(\lambda) & =\int_{0}^{\infty} \widetilde{f}(t) \cos t \sqrt{\lambda} d t, \\
\widetilde{f}(t) & =\frac{1}{\pi} \int_{0}^{\infty} f(\lambda) \cos t \sqrt{\lambda} \frac{d \lambda}{\sqrt{\lambda}} .
\end{aligned}
$$

Since $g \in L_{1}$ or, equivalently, $f \in L_{1}((0, \infty), d \lambda / \sqrt{\lambda})$, the formula for $\tilde{f}$ in fact holds pointwise. (The general theory only guarantees equality in $L_{2}$ sense.) Making the substitution $k=\sqrt{\lambda}$, we can write this as

$$
\widetilde{f}(t)=\frac{2}{\pi} \int_{0}^{\infty} g(k) \cos t k d k=\frac{1}{\pi} \int_{-\infty}^{\infty} g(k) e^{i t k} d k,
$$

where we have extended $g$ in the obvious way to all of $\mathbb{R}$. An inductive argument shows that then $g \in C^{\infty}(\mathbb{R})$ (the only issue being existence of the derivatives at zero). We may now integrate by parts $n+1$ times, and since $g^{(j)} \in L_{1}$ for all $j$, there are no boundary terms. Thus

$$
|\tilde{f}(t)| \leq \frac{1}{\pi t^{n+1}}\left\|g^{(n+1)}\right\|_{L_{1}(\mathbb{R})}=\frac{2}{\pi t^{n+1}}\left\|g^{(n+1)}\right\|_{L_{1}(0, \infty)} .
$$

Now break up $f$ as

$$
f(\lambda)=\int_{0}^{R} \widetilde{f}(t) \cos t \sqrt{\lambda} d t+\int_{R}^{\infty} \tilde{f}(t) \cos t \sqrt{\lambda} d t \equiv h(\lambda)+e(\lambda) .
$$

Again, the rapid decay of $\widetilde{f}$ ensures that everything holds pointwise for $\lambda \geq 0$. Moreover, writing $\rho(M)=\left\langle\varphi_{1}, E(M) \varphi_{2}\right\rangle$ for the spectral measure of $H$ and $\varphi_{1}, \varphi_{2}$, we have that

$$
\left\langle\varphi_{1}, h(H) \varphi_{2}\right\rangle=\int_{0}^{R} d t \tilde{f}(t) \int d \rho(\lambda) \cos t \sqrt{\lambda}=0,
$$

by Fubini's Theorem and Lemma 2.2(a). Therefore,

$$
\left|\left\langle\varphi_{1}, f(H) \varphi_{2}\right\rangle\right| \leq\left\|\varphi_{1}\right\|\left\|\varphi_{2}\right\| \sup _{\lambda \geq 0}|e(\lambda)|
$$

But the definition of the error term $e$ and (3.2) imply that

$$
|e(\lambda)| \leq \int_{R}^{\infty}|\widetilde{f}(t)| d t \leq \frac{2}{\pi n R^{n}}\left\|g^{(n+1)}\right\|_{L_{1}(0, \infty)},
$$

and the proof is complete. 
It is tempting to try to minimize over $n$ in the bounds from Theorems 3.2 , 3.3. This can certainly be done if one places some restrictions on the growth of the $L_{1}$ norms of the derivatives. We will pursue this theme in Sect. 5, where we will also compare Theorem 3.3 to the closely related work of Bouclet, Germinet, and Klein [1, 7]. These references use the Helffer-Sjöstrand formula and a Combes-Thomas estimate as their main tools.

\section{A PRiORi estimates on SPECTRAL MEASURES}

In a similar way, part (b) of Lemmas 2.1 and 2.2, respectively, yield results addressing the second problem mentioned above. This section is inspired by recent work of Germinet, Kiselev, and Tcheremchantsev [6. Lemma A.1]. We will take a look at this from the point of view suggested by the material of Section 2 . The treatment of [6] is again based on the Helffer-Sjöstrand formula and a Combes-Thomas estimate. In the one-dimensional case, we earlier discussed related problems in [13] from a point of view remotely reminiscent of the one taken here.

As in the previous section, we begin with the discrete case.

Theorem 4.1. Let $\varphi \in \ell_{2}\left(\mathbb{Z}^{d}\right)$, and let $V_{1}, V_{2}$ be two bounded potentials that agree on an $R$-neighborhood (with respect to the $|\cdot|_{1}$ norm on $\mathbb{Z}^{d}$ ) of the support of $\varphi$. Determine an interval $[a, b] \supset \sigma\left(H_{1}\right) \cup \sigma\left(H_{2}\right)$ and let $f \in C^{\infty}[a, b]$. Then, for $n=1,2, \ldots, 2 R$,

$$
\left|\left\langle\varphi, f\left(H_{1}\right) \varphi\right\rangle-\left\langle\varphi, f\left(H_{2}\right) \varphi\right\rangle\right| \leq 2\|\varphi\|^{2}\left\|f^{(n+1)}\right\|_{L_{1}(a, b)}\left(\frac{5(b-a)}{4 R}\right)^{n} .
$$

For an interesting application, let $f$ approximate the characteristic function of an interval $I$. Since $\left\langle\varphi, f\left(H_{j}\right) \varphi\right\rangle=\int f(\lambda) d \rho_{j}(\lambda)$, where $\rho_{j}$ is the spectral measure of $H_{j}$ and $\varphi$, Theorem 4.1 now says that it is possible to approximately compute $\rho(I)$ for an interval $I \subset \mathbb{R}$ with high accuracy, provided $V$ is known on an $R$ neighborhood of $\operatorname{supp} \varphi$ and $R|I| \gg 1$ (because $\left\|f^{(n+1)}\right\|_{1} \sim|I|^{-n}$ for typical approximations $f$ ). See [8, Sect. 8] for such an application of [6, Lemma A.1].

Proof. Let again

$$
s=\frac{2 \lambda-a-b}{b-a}, \quad g(s)=f\left(\frac{a+b}{2}+\frac{b-a}{2} s\right),
$$

and write, according to Theorem 3.1

$$
g(s)=p_{2 R}(s)+e_{2 R}(s), \quad-1 \leq s \leq 1,
$$

with a polynomial $p_{2 R}$ of degree $\leq 2 R$. By Lemma 2.1(b),

$$
\left\langle\varphi, p_{2 R}\left(\frac{2 H_{1}-a-b}{b-a}\right) \varphi\right\rangle=\left\langle\varphi, p_{2 R}\left(\frac{2 H_{2}-a-b}{b-a}\right) \varphi\right\rangle \text {. }
$$

As in the previous section, $\left\|g^{(n+1)}\right\|_{1}=\left(\frac{b-a}{2}\right)^{n}\left\|f^{(n+1)}\right\|_{1}$, so Theorem 3.1 shows that for $-1 \leq s \leq 1$,

$$
\left|e_{2 R}(s)\right| \leq\left(\frac{5(b-a)}{4 R}\right)^{n}\left\|f^{(n+1)}\right\|_{1},
$$

and the asserted inequality follows. 
Let us now discuss the continuous case. We will again assume that $H_{j} \geq 0$, which, of course, is just a normalization. Also, as noted in the previous section, the more natural variable is $k=\sqrt{\lambda}$ and thus our result reads as follows:

Theorem 4.2. Let $f \in C_{0}^{\infty}[0, \infty)$, and define $g(k)=f\left(k^{2}\right)$. Let $\varphi \in L_{2}\left(\mathbb{R}^{d}\right)$ and suppose that $V_{1}, V_{2}$ agree on an $R$-neighborhood of the support of $\varphi$. Then, for every $n \in \mathbb{N}$,

$$
\left|\left\langle\varphi, f\left(H_{1}\right) \varphi\right\rangle-\left\langle\varphi, f\left(H_{2}\right) \varphi\right\rangle\right| \leq \frac{4\|\varphi\|^{2}\left\|g^{(n+1)}\right\|_{L_{1}(0, \infty)}}{n \pi(2 R)^{n}} .
$$

Proof. The basic ideas are familiar by now, so it will suffice to provide a sketch of the argument. Expand $f$ :

$$
\begin{aligned}
& f(\lambda)=\int_{0}^{\infty} \tilde{f}(t) \cos t \sqrt{\lambda} d t, \\
& \widetilde{f}(t)=\frac{1}{\pi} \int_{-\infty}^{\infty} g(k) e^{i t k} d k .
\end{aligned}
$$

We argue as in the proof of Theorem 3.3, More specifically, write

$$
f(\lambda)=\int_{0}^{2 R} \widetilde{f}(t) \cos t \sqrt{\lambda} d t+\int_{2 R}^{\infty} \widetilde{f}(t) \cos t \sqrt{\lambda} d t \equiv h+e,
$$

note that $\left\langle\varphi,\left(h\left(H_{1}\right)-h\left(H_{2}\right)\right) \varphi\right\rangle=0$ by Lemma 2.2(b), and use (3.2) to bound the error term $e$.

\section{Some Remarks on Gevrey-type functions}

Usually, one defines the class of Gevrey functions on an interval $I \subset \mathbb{R}$ as follows (we restrict to the one-dimensional case right away because that is all we will need here): By definition, $f \in G_{s}(I)$ if $f \in C^{\infty}(I)$ and

$$
\left|f^{(n)}(x)\right| \leq C^{n+1} n^{s n} \quad\left(x \in K, n \in \mathbb{N}_{0}\right)
$$

for every compact subset $K \subset I$. Here, $s \geq 1$, and the constant $C$ may depend on $f$ and $K$. The functions $f \in G_{1}(I)$ are in fact real analytic (see [15, Theorem 19.9]). On the other hand, if $s>1$, then $G_{s}(I)$ contains compactly supported functions (easily constructed with the help of the functions $\exp \left(-x^{-b}\right)$ for suitable $b>0$ ).

For our purposes, it is clear from the results discussed in Sections 3 and 4 that global control on the derivatives is more relevant. This was also (and previously) recognized in [1]. The following definition seems most appropriate:

Definition 5.1. Let $I \subset \mathbb{R}$ be an interval and let $s \geq 1$. We say that $f \in G_{s}^{1}(I)$ if $f \in C^{\infty}(I)$ and there exists a constant $C>0$ so that

$$
\left\|f^{(n)}\right\|_{L_{1}(I)} \leq C^{n+1} n^{s n} \quad \text { for all } n \in \mathbb{N}_{0} .
$$

The relation of this to $G_{s}$ can be clarified by making the following quick observations: Suppose that $f \in G_{s}^{1}(I)$. Then there exists a constant $A>0$ (any positive $A$ with $A|I|>1$ will do), so that for every $n \in \mathbb{N}_{0}$, we can find an $x_{0}^{(n)} \in I$ so that 


$$
\begin{aligned}
& \left|f^{(n)}\left(x_{0}^{(n)}\right)\right| \leq A C^{n+1} n^{s n} \text {. But then for arbitrary } x \in I, \text { we have that } \\
& \qquad \begin{aligned}
\left|f^{(n)}(x)\right| & \leq\left|f^{(n)}\left(x_{0}^{(n)}\right)\right|+\left\|f^{(n+1)}\right\|_{L_{1}(I)} \leq A C^{n+1} n^{s n}+C^{n+2}(n+1)^{s(n+1)} \\
& =C^{n+1} n^{s n}\left(A+C(n+1)^{s}\left(1+\frac{1}{n}\right)^{s n}\right) .
\end{aligned}
\end{aligned}
$$

It follows that there exists a new constant $D$, independent of $n$, so that $\left|f^{(n)}(x)\right| \leq$ $D^{n+1} n^{s n}$ on $I$. In particular, $f \in G_{s}(I)$, but we have in fact obtained the stronger statement that the constants $C$ from (5.1) can be taken to be independent of $K$. We denote the set of functions satisfying such a uniform Gevrey condition by $G_{s}^{\text {unif }}(I)$. Also, it is obvious, by integrating the pointwise bounds, that $G_{s}(K) \subset G_{s}^{1}(K)$ for compact intervals $K \subset \mathbb{R}$. We have thus proved the following.

Proposition 5.1. (a) $G_{s}^{1}(I) \subset G_{s}^{\text {unif }}(I) \subset G_{s}(I)$;

(b) $G_{s}^{1}(K)=G_{s}(K)$ if $K \subset \mathbb{R}$ is a compact interval.

If our functions are in $G_{s}^{1}$, we can obtain more explicit information from the results of the preceding sections. We illustrate this with the Gevrey versions of Theorems 3.2 and 3.3, respectively. Of course, there are similar Gevreyzations of Theorems 4.1 and 4.2, which we will not make explicit.

Corollary 5.2. In the situation of Theorem [3.2, suppose that $f \in G_{s}^{1}[a, b]$. Then there exist constants $C, \gamma>0$ so that

$$
\left|\left\langle\delta_{x}, f(H) \delta_{y}\right\rangle\right| \leq C \exp \left(-\gamma|x-y|_{1}^{1 / s}\right) .
$$

Proof. It suffices to take $n \approx(R / C)^{1 / s}$ with a suitable constant $C$ in Theorem 3.2 , To spell this out more explicitly, first note that by Theorem 3.2 and the definition of $G_{s}^{1}$, we have that

$$
\left|\left\langle\delta_{x}, f(H) \delta_{y}\right\rangle\right| \leq\left(\frac{B n^{s}}{R}\right)^{n} \quad\left(n \in \mathbb{N}, R \equiv|x-y|_{1}>n\right)
$$

for a suitable constant $B>0$. We may assume that $B \geq 2 / e$, and we then pick $n$ so that

$$
\frac{R}{2 e B} \leq n^{s} \leq \frac{R}{e B}
$$

provided there actually exists an integer satisfying these bounds. This, however, will certainly be the case if $R$ is large enough. Since $s \geq 1, n$ is then not larger than $R-1$, and thus (5.2) shows that

$$
\left|\left\langle\delta_{x}, f(H) \delta_{y}\right\rangle\right| \leq e^{-n} \leq e^{-\gamma R^{1 / s}} .
$$

This proves the asserted bound for large $R$, and validity for all $R$ is then achieved by simply adjusting the constant $C$.

As observed above in Proposition [5.1(b), $G_{s}^{1}[a, b]=G_{s}[a, b]$, so we don't really need Definition 5.1 here. The class $G_{s}^{1}$ does become relevant, however, in the continuous case because then the spectra are unbounded and global bounds are needed. We have the following analog of Corollary 5.2.

Corollary 5.3. In the situation of Theorem 3.3 , suppose that $g \in G_{s}^{1}([0, \infty))$. Then there exist constants $C, \gamma>0$ so that

$$
\left|\left\langle\varphi_{1}, f(H) \varphi_{2}\right\rangle\right| \leq C\left\|\varphi_{1}\right\|\left\|\varphi_{2}\right\| \exp \left(-\gamma R^{1 / s}\right) .
$$


The proof is completely analogous to the proof of Corollary 5.2 .

In [1, Bouclet, Germinet, and Klein introduce the class of $C^{\infty}(I)$ functions $f$ that obey estimates of the form

$$
\int_{I}(1+|\lambda|)^{n-1}\left|f^{(n)}(\lambda)\right| d \lambda \leq C^{n+1} n^{s n},
$$

and they go on to prove that if this holds on an open interval $I$ containing the spectrum of $H$, then

$$
\left|\left\langle\varphi_{1}, f(H) \varphi_{2}\right\rangle\right| \lesssim \exp \left(-\gamma R^{(1 / s)-\epsilon}\right)
$$

for every $\epsilon>0$ [1, Theorem 1.4].

It may therefore be interesting to relate our hypothesis that $g \in G_{s}^{1}$, where $g(k)=f\left(k^{2}\right)$, to such a condition.

Theorem 5.4. Let $s \geq 1$. Suppose that $f \in C^{\infty}[0, \infty)$ and

$$
\int_{0}^{\infty}(1+\lambda)^{(n-1) / 2}\left|f^{(n)}(\lambda)\right| d \lambda \leq C^{n+1} n^{s n} \quad\left(n \in \mathbb{N}_{0}\right) .
$$

Then $g(k)=f\left(k^{2}\right) \in G_{s}^{1}([0, \infty))$.

Proof. This is elementary but a bit tedious. Working out the derivatives with the help of the chain and product rules, we see that $g^{(n)}$ is of the form

$$
g^{(n)}(k)=\sum a_{i j}(n) k^{i} f^{(j)}\left(k^{2}\right),
$$

where the sum ranges over all indices $n / 2 \leq j \leq n, 0 \leq i \leq j$ satisfying $i=2 j-n$. To confirm that this relation between $i$ and $j$ must hold, one can argue as follows: To produce a contribution of the form $k^{i} f^{(j)}$, we clearly must let precisely $j$ of the $n$ derivatives act on $f$. By the chain rule, this gives $j$ factors of $k$, and the remaining $n-j$ derivatives must then act on these. As a result, the exponent $j$ decreases by $n-j$; in other words, $i=2 j-n$. The other restrictions on $i, j$ follow from this.

Our task is to extract some information on the coefficients $a_{i j}(n)$. By taking the derivative in the above representation, it follows that the $a_{i j}(n)$ obey the recursion

$$
a_{i j}(n)=(i+1) a_{i+1, j}(n-1)+2 a_{i-1, j-1}(n-1) .
$$

It will be convenient to use the difference $d=j-i$ as the parameter indexing the coefficients $a$. Note that for fixed $n$, we have that $0 \leq d \leq n / 2$. Moreover, $a_{i j}(n)$ can be different from zero only if $i=n-2 d, j=n-d$. We'll use the abbreviation $C_{d}(n)=a_{n-2 d, n-d}(n)$, and we then claim that

$$
0 \leq C_{d}(n) \leq \frac{2^{n-2 d} n^{2 d}}{d !} .
$$

Of course, only the upper bound needs proof, and we can use induction on $d$. The case $d=0$ corresponds to letting all the derivatives act on $f$, so $C_{0}(n)=2^{n}$. Now assume that (5.4) holds for $d-1$, with $d \geq 1$. By (5.3) and the definition of $C_{d}(n)$,

$$
C_{d}(n)=(n-2 d+1) C_{d-1}(n-1)+2 C_{d}(n-1) .
$$


We will now iterate this, using the induction hypothesis and the fact that $d \geq 1$ to estimate the first term on the right-hand side. In the first step, we obtain

$$
\begin{aligned}
C_{d}(n) & \leq \frac{2^{n-2 d+1}}{(d-1) !}(n-1)^{2 d-1}+2 C_{d}(n-1) \\
& =\frac{2^{n-2 d+1}}{(d-1) !}(n-1)^{2 d-1}+2((n-1)-2 d+1) C_{d-1}(n-2)+2^{2} C_{d}(n-2) \\
& \leq \frac{2^{n-2 d+1}}{(d-1) !}\left((n-1)^{2 d-1}+(n-2)^{2 d-1}\right)+2^{2} C_{d}(n-2) .
\end{aligned}
$$

Continuing in this way and recalling that $C_{d}(n)=0$ as soon as $n<2 d$, we see that

$$
C_{d}(n) \leq \frac{2^{n-2 d+1}}{(d-1) !} \sum_{j=1}^{n-1} j^{2 d-1} \leq \frac{2^{n-2 d+1}}{(d-1) !} \int_{1}^{n} x^{2 d-1} d x<\frac{2^{n-2 d} n^{2 d}}{d !}
$$

as required.

We are now ready to estimate the integrals $\int_{0}^{\infty}\left|g^{(n)}\right| d k$. To prove Theorem 5.4, it clearly suffices to show that

$$
C_{d}(n) \int_{0}^{\infty} k^{n-2 d}\left|f^{(n-d)}\left(k^{2}\right)\right| d k \leq C^{n+1} n^{s n}
$$

for $0 \leq d \leq n / 2$, with a constant $C$ independent of $d$ and $n$. We will split these integrals into two parts, corresponding to $0 \leq k \leq 1$ and $k>1$, respectively. Consider first $C_{d}(n) \int_{1}^{\infty} \cdots$. Using $\lambda=k^{2}$ as the variable, we can write this as

$$
\frac{1}{2} C_{d}(n) \int_{1}^{\infty} \lambda^{(n-2 d-1) / 2}\left|f^{(n-d)}(\lambda)\right| d \lambda,
$$

and, by hypothesis, we have a bound of the form $C_{d}(n) C^{n+1}(n-d)^{s(n-d)}$. So, taking (5.4) into account, we must now show that there exists a constant $C>0$ so that

$$
\frac{n^{2 d}}{d^{d}}(n-d)^{s(n-d)} \leq C^{n+1} n^{s n}
$$

for all $0 \leq d \leq n / 2$. This can be done quite easily, by simply further estimating $(n-d)^{s(n-d)} \leq n^{s(n-d)}$.

A similar argument lets us bound $C_{d}(n) \int_{0}^{1} \cdots$. We can in fact work with pointwise bounds. Indeed, by hypothesis and Proposition 5.1(b), $f \in G_{s}[0,1]$, so, reasoning along the above lines, we see that $g \in G_{s}[0,1]$, too.

\section{ACKNOWLEDGMENTS}

I thank Wilhelm Schlag for showing me the proof of Lemma2.3 and Adam Sikora for informing me of his work.

\section{REFERENCES}

[1] J.-M. Bouclet, F. Germinet, and A. Klein, Sub-exponential decay of operator kernels for functions of generalized Schrödinger operators, Proc. Amer. Math. Soc. 132 (2004), 27032712. MR 2054797 (2005c:35059)

[2] J. Cheeger, M. Gromov, and M. Taylor, Finite propagation speed, kernel estimates for functions of the Laplace operator, and the geometry of complete Riemannian manifolds, J. Differential Geom. 17 (1982), 15-53. MR658471 (84b:58109)

[3] E.W. Cheney, Introduction to Approximation Theory, McGraw-Hill, New York 1966. MR0222517(36:5568) 
[4] E.A. Coddington and N. Levinson, Theory of Ordinary Differential Equations, McGraw-Hill, New York 1972. MR0069338(16:1022b)

[5] L.C. Evans, Partial Differential Equations, Graduate Studies in Mathematics, Vol. 19, American Mathematical Society, Rhode Island 1998. MR.1625845 (99e:35001)

[6] F. Germinet, A. Kiselev, and S. Tcheremchantsev, Transfer matrices and transport for Schrödinger operators, Ann. Inst. Fourier 54 (2004), 787-830. MR2097423 (2005h:81123)

[7] F. Germinet and A. Klein, Operator kernel estimates for functions of generalized Schrödinger equations, Proc. Amer. Math. Soc. 131 (2002), 911-920. MR1937430 (2003k:47067)

[8] A. Kiselev, Imbedded singular continuous spectrum for Schrödinger operators, J. Amer. Math. Soc. 18 (2005), 571-603. MR2138138 (2005m:34203)

[9] P. Lax and R. Phillips, Scattering theory, 2nd edition, Academic Press, Boston 1989. MR.1037774 (90k:35005)

[10] M. Reed and B. Simon, Methods of Modern Mathematical Physics, I. Functional Analysis, Academic Press, New York 1972. MR0493419 (58:12429a)

[11] M. Reed and B. Simon, Methods of Modern Mathematical Physics, III. Scattering Theory, Academic Press, New York 1979. MR529429 (80m:81085)

[12] C. Remling, Schrödinger operators and de Branges spaces, J. Funct. Anal. 196 (2002), 323394. MR1943095 (2003j:47055)

[13] C. Remling, Universal bounds on spectral measures for one-dimensional Schrödinger operators, J. Reine Angew. Math. 564 (2003), 105-117. MR2021036 (2005f:47109)

[14] T.J. Rivlin, An Introduction to the Approximation of Functions, Blaisdell Publishing, Waltham 1969. MR0249885(40:3126)

[15] W. Rudin, Real and Complex Analysis, 3rd edition, McGraw-Hill, New York 1987. MR924157 (88k:00002)

[16] A. Sikora, On-diagonal estimates on Schrödinger semigroup kernels and reduced heat kernels, Commun. Math. Phys. 188 (1997), 233-249. MR.1471338(98k:58213)

[17] B. Simon, Schrödinger operators in the twentieth century, J. Math. Phys. 41 (2000), 35233555. MR1768631(2002d:81060)

[18] A.F. Timan, Theory of Approximation of Functions of a Real Variable, Pergamon Press, Oxford 1963. MR0192238 (33:465)

Department of Mathematics, University of Oklahoma, Norman, Oklahoma 73019-0315

E-mail address: cremling@math.ou.edu

$U R L$ : www.math.ou.edu/ cremling 